\title{
A comparison of twice-daily exenatide and biphasic insulin aspart in patients with type 2 diabetes who were suboptimally controlled with sulfonylurea and metformin: a non-inferiority study
}

\author{
M. A. Nauck • S. Duran • D. Kim • D. Johns • \\ J. Northrup • A. Festa $・$ R. Brodows $・$ M. Trautmann
}

Received: 29 June 2006 / Accepted: 18 September 2006 / Published online: 8 December 2006

(C) Springer-Verlag 2006

\author{
Abstract \\ aspart. \\ $0510-2$ and is accessible to authorised users. \\ M. A. Nauck $(\bowtie)$ \\ Diabetes Center, \\ Kirchberg 21, \\ 37431 Bad Lauterberg im Harz, Germany \\ e-mail: nauck@diabeteszentrum.de \\ S. Duran \\ Department of Endocrinology, Valme Hospital, \\ Seville, Spain \\ D. Kim \\ Amylin Pharmaceuticals, \\ San Diego, CA, USA \\ D. Johns $\cdot$ J. Northrup $\cdot$ R. Brodows \\ Lilly Research Laboratories, \\ Indianapolis, IN, USA
A. Festa
Lilly Research Laboratories,
Vienna, Austria
M. Trautmann
Lilly Research Laboratories,
Hamburg, Germany

Aims/hypothesis The aim of this 52-week, open-label, noninferiority trial was to compare the safety and efficacy of exenatide (an incretin mimetic) with that of biphasic insulin

Materials and methods Patients on metformin and a sulfonylurea were randomised to exenatide $(n=253 ; 5 \mu \mathrm{g}$ twice daily for 4 weeks, $10 \mu \mathrm{g}$ thereafter) or biphasic insulin aspart ( $n=248$; twice-daily doses titrated for optimal

Electronic supplementary material A list of the site investigators is available as electronic supplementary material in the online version of this article at http://dx.doi.org/10.1007/s00125-006- glucose control), while continuing with metformin and sulfonylurea treatment.

Results Glycaemic control achieved with exenatide was non-inferior to that achieved with biphasic insulin aspart (mean $\pm \mathrm{SEM}, \mathrm{HbA}_{1 \mathrm{c}}$ change: exenatide $-1.04 \pm 0.07 \%$, biphasic insulin aspart $-0.89 \pm 0.06 \%$; difference -0.15 [95\% CI -0.32 to 0.01$] \%)$. Exenatide-treated patients lost weight, while patients treated with biphasic insulin aspart gained weight [between-group difference -5.4 (95\% CI -5.9 to -5.0$) \mathrm{kg}$ ]. Both treatments reduced fasting serum glucose (exenatide $-1.8 \pm 0.2 \mathrm{mmol} / \mathrm{l}, p<0.001$; biphasic insulin aspart $-1.7 \pm 0.2 \mathrm{mmol} / 1, p<0.001)$. Greater reductions in postprandial glucose excursions following morning $(p<0.001)$, midday $(p=0.002)$ and evening meals $(p<0.001)$ were observed with exenatide. The withdrawal rate was $21.3 \%(54 / 253)$ for exenatide and $10.1 \%(25 / 248)$ for biphasic insulin aspart. Nausea (33\% incidence, 3.5\% discontinuation) was the most common adverse event observed with exenatide.

Conclusions/interpretation Exenatide treatment resulted in $\mathrm{HbA}_{1 \mathrm{c}}$ reduction similar to biphasic insulin aspart and provided better postprandial glycaemic control, making it a potential alternative for the treatment of type 2 diabetes. Treatment with biphasic insulin aspart was associated with weight gain and lower risk of adverse gastrointestinal events. Although the availability of glucose-lowering agents associated with weight reduction may be considered a therapeutic advance, the long-term implications of progressive weight reduction observed with exenatide have yet to be defined.

Keywords Biphasic insulin aspart · Exenatide . Incretin mimetic · Non-inferiority · Type 2 diabetes . Weight reduction 


\begin{tabular}{|c|c|}
\hline \multicolumn{2}{|c|}{ Abbreviations } \\
\hline GLP-1 & glucagon-like peptide-1 \\
\hline HOMA & homeostasis model assessment \\
\hline HOMA-B & $\begin{array}{l}\text { homeostasis model assessment of beta cell } \\
\text { function }\end{array}$ \\
\hline HOMA-S & $\begin{array}{l}\text { homeostasis model assessment of insulin } \\
\text { sensitivity }\end{array}$ \\
\hline ITT & intention-to-treat \\
\hline MMRM & mixed model repeated measures \\
\hline SMBG & self-monitored blood glucose \\
\hline
\end{tabular}

\section{Introduction}

Incretin mimetics are an emerging class of compounds that have the ability to improve glycaemic control through multiple antihyperglycaemic actions similar to natural incretin hormones [1-3]. Exenatide, a first-in-class incretin mimetic, shares several glucoregulatory actions with the incretin glucagon-like peptide-1 (GLP-1) including enhancement of glucose-dependent insulin secretion, suppression of inappropriately elevated postprandial glucagon secretion, reduction of food intake, and slowing of gastric emptying $[4,5]$. In addition, both GLP-1 and exenatide have been shown to enhance beta cell mass in animal models $[6,7]$. In 30-week placebo-controlled studies [8-10], their open-label uncontrolled extensions [11], and a 26-week comparator study vs insulin glargine [12], exenatide-treated patients with type 2 diabetes that was suboptimally controlled with oral glucoselowering agents demonstrated significantly improved glycaemic control with the additional benefit of sustained weight reduction. The US Food and Drug Administration has approved exenatide for the treatment of type 2 diabetes in adult patients who have not achieved adequate glycaemic control and who are taking metformin, a sulfonylurea or a combination of both.

To date, no studies have compared the effects of exenatide with an insulin analogue formulation designed to provide both basal and postprandial glycaemic control. The current study was thus designed to compare the safety and efficacy of exenatide with that of biphasic insulin aspart 30/70 in patients with type 2 diabetes, who were failing to reach treatment goals with optimally effective doses of metformin and a sulfonylurea.

\section{Subjects and methods}

Study design Investigators and patients from 13 countries participated in this industry-initiated study between November 2003 and April 2005. A non-inferiority design was chosen because biphasic insulin aspart (30\% rapid-acting insulin aspart) is a well-matched comparator for exenatide (both agents are injected twice daily and influence fasting and postprandial glycaemia), although biphasic insulin aspart, unlike exenatide, can be continually titrated to desired levels. It was estimated that 446 patients would provide $90 \%$ power to test the hypothesis that exenatide was non-inferior to biphasic insulin aspart with regard to changes in $\mathrm{HbA}_{1 \mathrm{c}}$. The non-inferiority margin for the difference in $\mathrm{HbA}_{1 \mathrm{c}}$ change between treatments was predefined as $0.4 \%$, with non-inferiority demonstrated by excluding the $0.4 \%$ non-inferiority margin with the upper limit of a two-sided $95 \%$ CI for the mean difference between treatments. The margin of $0.4 \%$ was selected on the assumption that $\mathrm{HbA}_{1 \mathrm{c}}$ differences of less that $0.3 \%$ are of questionable clinical relevance and that the benefit of weight reduction may account for an additional $0.1 \%$ of $\mathrm{HbA}_{1 \mathrm{c}}$ difference.

Patients meeting study entry criteria were randomly assigned (with equal probability) to receive either exenatide or biphasic insulin aspart (henceforth referred to as premixed insulin) according to a computer-generated randomisation table administered by the sponsor via an automated voiceresponse system. Randomisation was stratified by site and patients were equally distributed across treatments within two strata based on screening values of $\mathrm{HbA}_{1 \mathrm{c}}(\leq 9.0$ and $>9.0 \%$ ). Patients assigned to the exenatide group used a multi-use pen to subcutaneously inject (within $15 \mathrm{~min}$ before morning and evening meals) a fixed dose of $5 \mu \mathrm{g}$ twice daily for 4 weeks and $10 \mu \mathrm{g}$ twice daily for the remainder of the study. If frequent nausea developed (daily episodes for $>1$ week duration), patients had the option to decrease their dose to $5 \mu \mathrm{g}$ twice daily. Patients in the comparator group subcutaneously injected premixed insulin before the morning and evening meals. Investigators chose the starting insulin dose for patients following randomisation, and contacted patients at regular intervals to discuss glycaemic control. A forced titration schedule was not used in this trial. Investigators were instructed to adjust insulin doses to achieve an optimal balance between glycaemic control and risk of hypoglycaemia as dictated by best clinical practice. The decision to adjust insulin therapy, and the mode of patient empowerment to self-adjust insulin doses, was ultimately left up to each investigator's clinical judgement. Multiple options were available to guide intensification of insulin therapy including: (1) ongoing analysis of the patient's diary and home glucose monitoring results; and (2) a titration guideline outlining minimum targets for fasting glucose $(<7 \mathrm{mmol} / \mathrm{l}[126 \mathrm{mg} / \mathrm{dl}])$ and 2-h postprandial glucose $(<10 \mathrm{mmol} / 1[180 \mathrm{mg} / \mathrm{dl}])$. In addition, the importance of optimising insulin doses was discussed with investigators at both study initiation and mid-study meetings where they were encouraged to optimise glucose control by titrating insulin doses as high as clinically 
possible. Patients entering this study maintained optimally effective prestudy metformin and sulfonylurea dosages; if hypoglycaemia events occurred, investigators reduced the sulfonylurea dose by approximately $50 \%$ for patients on exenatide or adapted the insulin dose for patients on insulin.

Study participants Patients were recruited according to local practices and through advertising, and all participants gave written informed consent before participation. Investigators were compensated for performing study procedures and collecting data as defined by the study protocol. Patients in both treatment groups were reimbursed for travel costs and time. Neither investigators nor patients were offered any additional incentives for participation in the trial. Eligible patients were between 30 and 75 years of age and had suboptimal glycaemic control despite receiving optimally effective metformin and sulfonylurea therapy for at least 3 months. Inclusion criteria included, at the time of screening, $\mathrm{HbA}_{1 \mathrm{c}}$ levels $\geq 7.0$ and $\leq 11.0 \%$, a $\mathrm{BMI} \geq 25$ and $\leq 40 \mathrm{~kg} / \mathrm{m}^{2}$, and a history of stable body weight $(\leq 10 \%$ variation for $\geq 3$ months). Patients were excluded if they: (1) had had more than three episodes of severe hypoglycaemia within 6 months prior to screening; (2) had used any prescription drug to promote weight loss within 3 months; or (3) had been treated with insulin, thiazolidinediones, alpha-glucosidase inhibitors or meglitinides for longer than 2 weeks within 3 months. A complete list of inclusion and exclusion criteria can be found at the online clinical trial registry ClinicalTrials.gov (study identifier: NCT00082407).

Study measurements A common clinical protocol was approved by institutional review boards at each site and the study was conducted in accordance with the principles described in the Declaration of Helsinki. $\mathrm{HbA}_{1 \mathrm{c}}$ levels were measured at screening, randomisation (baseline, week 0), and at weeks 12, 16, 28, 40 and 52 (or early discontinuation). Blood chemistry was assessed at screening and week 52 (or early discontinuation). Fasting serum glucose and fasting serum lipids were assessed at baseline, and at weeks 16 and 52 (or early discontinuation). Patients performed two separate self-monitored blood glucose (SMBG) profiles during the 2 weeks prior to study baseline and at weeks 4 , 12, 28 and 52. Patients collected SMBG measurements using commercially available blood glucose meters-just before each meal (prebreakfast, prelunch, presupper), $2 \mathrm{~h}$ after each meal (postbreakfast, postlunch, postsupper), and at 03.00 hours. Postprandial blood glucose excursions were calculated on the basis of the difference between preprandial and postprandial measurements taken at morning, midday and evening meals. Homeostasis model assessment (HOMA) $[13,14]$ of beta cell function (HOMA-B) and insulin sensitivity (HOMA-S) were generated from fasting serum glucose and C-peptide levels obtained at baseline prior to exenatide administration and an endpoint at least $12 \mathrm{~h}$ after the previous dose. Since exogenous insulin might suppress C-peptide levels used in the HOMA calculations [14], beta cell function and insulin sensitivity were only assessed in the exenatide-treated patients. Fasting glucose levels were measured using the hexokinase enzymatic method (BMD Hitachi analyser; Roche Diagnostics, Indianapolis, IN, USA; intra-assay CV 2.0 to $2.2 \%$ ). Fasting C-peptide levels were measured using the chemiluminescent immunoassay method (DPC Immulite 2000; Diagnostics Product Corporation, Hollistion, MA, USA; intra-assay CV 3.4 to $5.3 \%$ ).

Adverse events were assessed at each visit. A hypoglycaemic episode was defined as any time a patient experienced a sign or symptom of hypoglycaemia or noted a blood glucose level $<3.4 \mathrm{mmol} / 1(60 \mathrm{mg} / \mathrm{dl})$ during selfmonitoring, whether or not this level was associated with signs, symptoms or treatment. The severity (mild, moderate or severe) and timing (nocturnal or daytime) of each hypoglycaemic event and whether it could be attributed to therapy (yes or no) were assessed by the investigator. Plasma anti-exenatide antibodies were measured at baseline and final visit, as previously described [15].

Statistical analysis Two analysis sets were predefined for the analysis of $\mathrm{HbA}_{1 \mathrm{c}}$ : (1) an intention-to-treat (ITT) sample, defined as patients who received at least one dose of study medication and had at least one post-baseline measurement of $\mathrm{HbA}_{1 \mathrm{c}}$; and (2) a per-protocol sample, defined as patients who had at least 12 weeks of exposure to study medication and no violations of screening criteria or discontinuation criteria. In non-inferiority trials, non-ITT analyses provide protection from type 1 error risk, and greater confidence is evoked when conclusions from ITT and per-protocol datasets are consistent [16]. SAS software (SAS Institute, Cary, NC, USA) was used to conduct all statistical analyses.

Primary efficacy analyses were based on a mixed model repeated measures (MMRM) analysis of covariance with $\mathrm{HbA}_{1 \mathrm{c}}$ as the dependent variable and treatment, baseline $\mathrm{HbA}_{1 \mathrm{c}}$, country, week of visit, and treatment-by-week interaction as fixed effects, and patient and error as random effects. Compound symmetry was used to model the covariance structure within patients. All post-baseline measurements of the change in $\mathrm{HbA}_{1 \mathrm{c}}$ were included in the analysis with no imputations of missing data. Using SAS PROC MIXED, we obtained least-squares estimates of the treatment difference and standard error from the MMRM model to compute the two-sided $95 \%$ CI used to test non-inferiority. Treatment comparisons were conducted at each scheduled visit; however, for the purpose of establishing non-inferiority, inferences were based on the 
estimate of the treatment difference at week 52. Analyses of fasting serum glucose and body weight were also conducted using MMRM models. Additional continuous secondary measures (e.g. fasting serum lipids, SMBG levels, hypoglycaemia rates) were analysed using a model that included treatment, country and baseline value of the dependent variable as covariates. Tests of treatment effects were conducted at a two-sided significance level of 0.05 , and all 95\% CIs for the difference between treatments were calculated on the basis of exenatide minus insulin. Fisher's exact tests were used for comparisons based on categorical variables (e.g. adverse events, incidence of hypoglycaemia). Predefined subgroup analyses were completed to determine the influence of baseline characteristics, sulfonylurea dose reduction, and antibody status on changes in $\mathrm{HbA}_{1 \mathrm{c}}$ and fasting serum glucose. Two subgroup analyses were added to the statistical plan (post-hoc), including an evaluation of the association between weight reduction and gastrointestinal adverse events and an evaluation of the association between sulfonylurea dose reduction and hypoglycaemia rates.

\section{Results}

Patient disposition and clinical characteristics Of the 641 patients screened, 501 met criteria for inclusion in the ITT sample and 446 completed at least 12 weeks of the study (per-protocol sample) (Fig. 1). The withdrawal rate was $21.3 \%$ (54/253) for exenatide and 10.1\% (25/248) for biphasic insulin aspart. The most common reasons for early discontinuation were protocol violations followed by adverse events. A greater proportion of patients in the exenatide-treated group (20/253) withdrew from the study because of adverse events compared with the premixed insulin group (0/248).

At baseline (Table 1), participants were generally overweight (BMI $30.4 \pm 4.1 \mathrm{~kg} / \mathrm{m}^{2}$ ) with a long history of diabetes (duration $10 \pm 6$ years) and suboptimal glycaemic control $\left(\mathrm{HbA}_{1 \mathrm{c}} 8.6 \pm 1.0 \%\right)$. At the end of the study, $80 \%$ $(195 / 245)$ of exenatide-treated patients were using the $10 \mu \mathrm{g}$ twice-daily dose. The mean dose of premixed insulin increased from $15.7 \pm 9.5 \mathrm{U} /$ day at week 2 to $24.4 \pm 15.6$ U/day at week 52 . Approximately $33 \%$ (84/253) of exenatide-
Fig. 1 Study profile of patients randomised to receive exenatide or premixed insulin

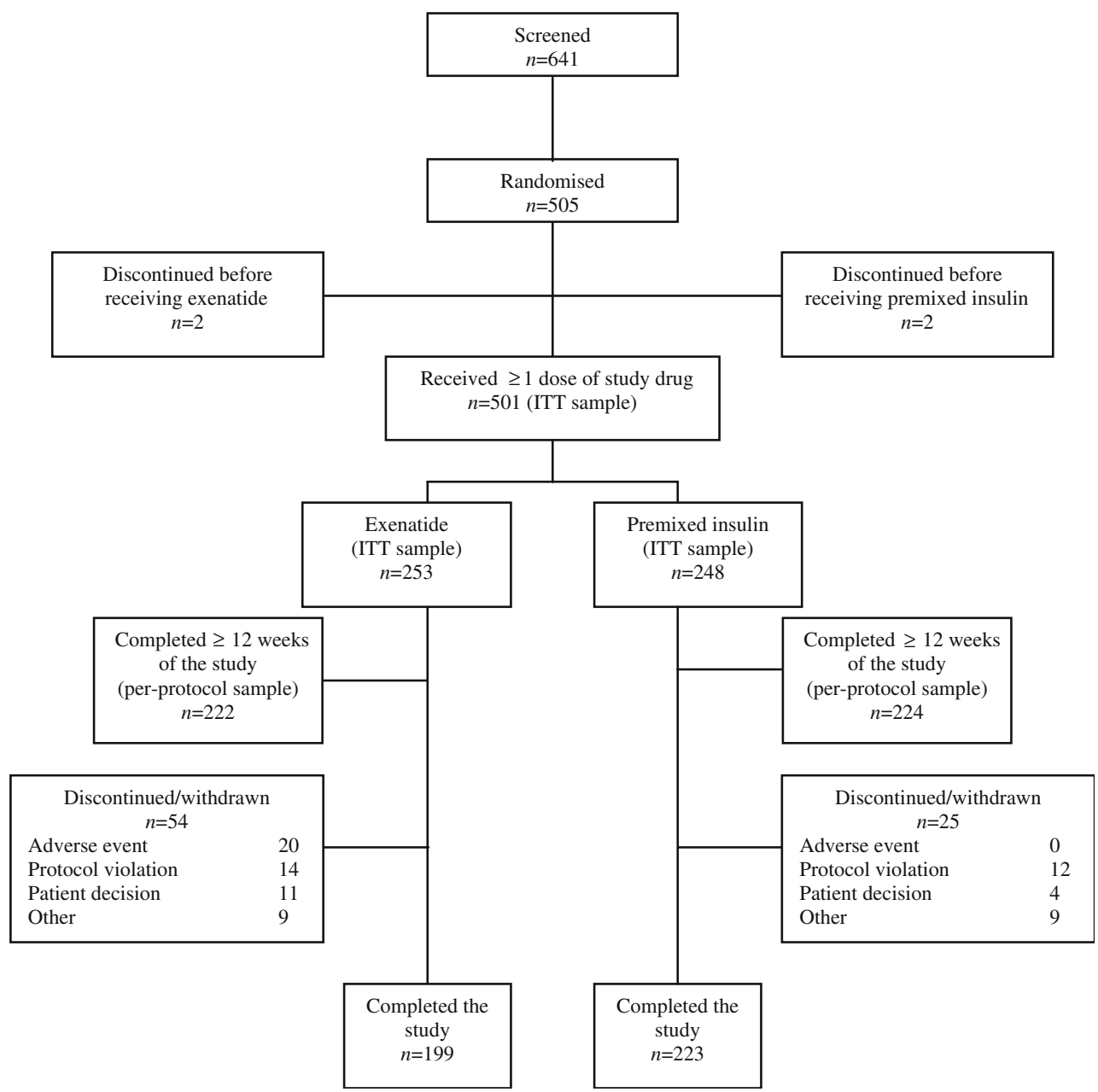


Table 1 Baseline demographic and metabolic characteristics of randomised patients (intention to treat sample)

\begin{tabular}{lll}
\hline Characteristics & $\begin{array}{l}\text { Exenatide } \\
(n=253)\end{array}$ & $\begin{array}{l}\text { Premixed insulin } \\
(n=248)\end{array}$ \\
\hline Age (years) & $59(9)$ & $58(9)$ \\
Sex, women (\%) & 47 & 51 \\
Body weight (kg) & $85.5(15.7)$ & $83.4(15.6)$ \\
BMI $\left(\mathrm{kg} / \mathrm{m}^{2}\right)$ & $30.6(4.0)$ & $30.2(4.2)$ \\
Fasting serum glucose (mmol/l) & $11.0(2.7)$ & $11.3(2.8)$ \\
HbA $_{1 \mathrm{c}}(\%)$ & $8.6(1.0)$ & $8.6(1.1)$ \\
Duration of diabetes (years) & $9.8(6.3)$ & $10.0(6.2)$ \\
HDL (mmol/l) & $1.2(0.3)$ & $1.2(0.3)$ \\
LDL (mmol/l) & $3.0(0.9)$ & $2.9(0.9)$ \\
Total cholesterol (mmol/l) & $5.1(1.0)$ & $5.0(1.0)$ \\
Triacylglycerol (mmol/l) & $2.2(2.0)$ & $2.2(1.5)$ \\
Systolic blood pressure (mmHg) & $138(16)$ & $136(15)$ \\
Diastolic blood pressure (mmHg) & $81(10)$ & $80(10)$ \\
\hline
\end{tabular}

Characteristics are presented as mean (SD); sex is presented a raw percentage value

treated patients and 5\% (12/248) of patients treated with premixed insulin had their sulfonylurea dose reduced during the study. During the 1-year study period, lipidlowering therapies were administered to approximately $25 \%(64 / 253)$ of exenatide patients and approximately $25 \%(63 / 248)$ of premixed insulin patients. Antihypertensive therapies were administered to approximately $40 \%$ $(102 / 253)$ of exenatide patients and 33\% (83/248) of premixed insulin patients.

Glycaemic control The change in $\mathrm{HbA}_{1 \mathrm{c}}$ with time is illustrated in Fig. 2, with additional key efficacy results shown in Table 2. The upper limit of the CI, representing the difference between treatments in change in $\mathrm{HbA}_{1 \mathrm{c}}$, was below the prespecified non-inferiority margin of $0.4 \%$ using both the ITT and per-protocol datasets. Therefore, the primary objective of the study was achieved, with exenatide demonstrating non-inferiority to premixed insulin with respect to change in $\mathrm{HbA}_{1 \mathrm{c}}$. In addition, both exenatide- and premixed insulin-treated patients exhibited significant reductions in fasting serum glucose levels from baseline to week 52 (Table 2). Accounting for $\mathrm{HbA}_{1 \mathrm{c}}$ stratification at screening, the between-group difference for achievement of a target $\mathrm{HbA}_{1 \mathrm{c}} \leq 7.0 \%$ was statistically significant (exenatide $32 \%$ [72/227], premixed insulin 24\% [57/237]; between-group $p=0.038$ ). Baseline demographic characteristics (i.e. sex, age and $\mathrm{BMI}$ ) were not significantly associated with changes in $\mathrm{HbA}_{1 \mathrm{c}}$ and fasting serum glucose. Observed reductions in $\mathrm{HbA}_{1 \mathrm{c}}$ were similar in exenatide-treated patients with stable and reduced sulfonylurea doses (descriptive mean \pm SD change: $-0.99 \pm 1.31 \% ;-0.93 \pm 1.13 \%$, respectively).

SMBG profiles (Fig. 3) indicated significantly reduced endpoint glucose levels at all time points in both treatment groups (all within-group values, $p<0.001$ ). At week 52 , the premixed insulin group had significantly lower mean glucose values at prebreakfast $(p=0.037)$, prelunch $(p=0.004)$ and 03.00 hours $(p=0.002)$. Conversely, exenatide-treated patients had lower 2-h postbreakfast $(p<0.001)$ and postsupper $(p<0.001)$ blood glucose concentrations. In addition, exenatide-treated patients had significantly greater reductions in postprandial glucose excursions (the difference between preprandial and postprandial levels) following the morning $(p<0.001)$, midday $(p<0.002)$ and evening meals $(p<0.001)$ than did patients treated with premixed insulin.

Body weight Mean $( \pm \mathrm{SD})$ baseline body weights were $86 \pm$ $16 \mathrm{~kg}$ and $83 \pm 16 \mathrm{~kg}$ for patients in the exenatide and premixed insulin groups, respectively. Exenatide-treated patients experienced a steady decline in body weight, while those receiving premixed insulin gained weight throughout the trial (Fig. 2). A statistically significant between-group difference in body weight was observed as early as week 2 and progressed to a difference of $-5.5 \pm 0.2 \mathrm{~kg}$ (leastsquares mean $\pm \mathrm{SEM}, 95 \% \mathrm{CI}-5.9$ to $-5.0 \mathrm{~kg} ; p<0.001)$ at week 52. A small non-significant difference in magnitude of weight reduction was seen between exenatide-treated patients who experienced at least one episode of nausea or
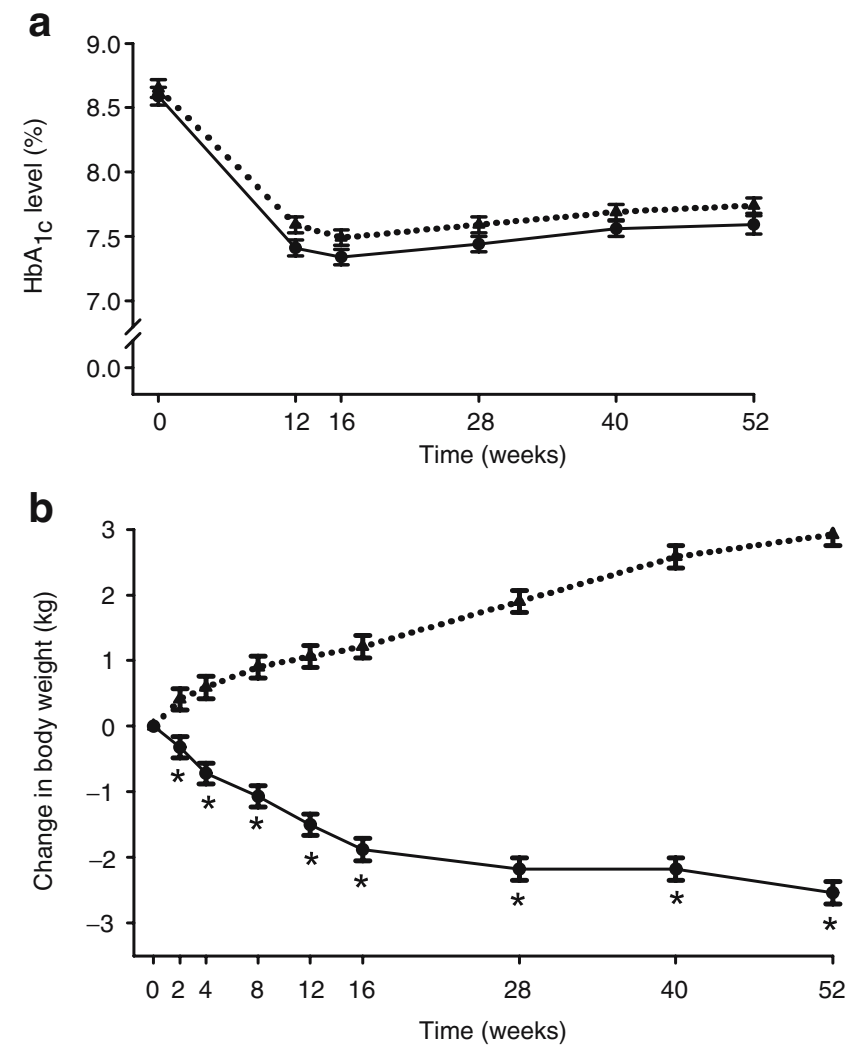

Fig. 2 Changes in $\mathrm{HbA}_{1 \mathrm{c}}$ (a) and body weight (b). Time course for $\mathrm{HbA}_{1 \mathrm{c}}$ level and body weight from week 0 to week 52 for the exenatide group (circles) compared with the premixed insulin group (triangles; ITT sample). Values are mean (SEM). ${ }^{*} p<0.001$ compared with premixed insulin measured at the same time point 
Table 2 Mean changes in $\mathrm{HbA}_{1 \mathrm{c}}$, fasting serum glucose and body weight

\begin{tabular}{|c|c|c|c|}
\hline Variable & $\begin{array}{l}\text { Exenatide } \\
\text { Mean }(\mathrm{SEM})^{\mathrm{a}}\end{array}$ & $\begin{array}{l}\text { Premixed insulin } \\
\text { Mean }(\mathrm{SEM})^{\mathrm{a}}\end{array}$ & $\begin{array}{l}\text { Exenatide-insulin } \\
\text { (95\% CI, } p \text { value) }\end{array}$ \\
\hline \multicolumn{4}{|l|}{ ITT sample $(n=501)$} \\
\hline$\Delta \mathrm{HbA}_{1 \mathrm{c}} \%$ from baseline to week $52, \%$ points & $-1.04(0.07)^{*}$ & $-0.89(0.06)^{*}$ & $-0.15(-0.32$ to $0.01, p=0.067)$ \\
\hline$\Delta$ Fasting serum glucose $(\mathrm{mmol} / \mathrm{l})$ from baseline to week 52 & $-1.8(0.2)^{*}$ & $-1.7(0.2)^{*}$ & $-0.1(-0.6$ to $0.4, p=0.689)$ \\
\hline$\Delta$ Body weight $(\mathrm{kg})$ from baseline to week 52 & $-2.5(0.2)^{*}$ & $+2.9(0.2)^{*}$ & $-5.4(-5.9$ to $-5.0, p<0.001)$ \\
\hline \multicolumn{4}{|l|}{ Per-protocol sample $(n=446)$} \\
\hline$\Delta \mathrm{HbA}_{1 \mathrm{c}} \%$ from baseline to week $52, \%$ points & $-1.04(0.07)^{*}$ & $-0.89(0.06)^{*}$ & $-0.15(-0.32$ to $0.01, p=0.072)$ \\
\hline$\Delta$ Fasting serum glucose $(\mathrm{mmol} / \mathrm{l})$ from baseline to week 52 & $-1.8(0.2)^{*}$ & $-1.7(0.2)^{*}$ & $-0.1(-0.6$ to $0.4, p=0.656)$ \\
\hline$\Delta$ Body weight $(\mathrm{kg})$ from baseline to week 52 & $-2.5(0.2)^{*}$ & $+2.9(0.2)^{*}$ & $-5.4(-5.9$ to $-5.0, p<0.001)$ \\
\hline
\end{tabular}

${ }^{*} p<0.001$ for baseline to week 52 within-group change

${ }^{a}$ Least-squares mean changes were obtained from a mixed model repeated-measures analysis of covariance

vomiting $(-2.7 \pm 3.2 \mathrm{~kg}, n=94)$ and patients who did not report any nausea or vomiting $(-2.1 \pm 3.4 \mathrm{~kg}, n=157)$.

Beta cell function and insulin sensitivity Exenatide patients exhibited a statistically significant baseline-to-endpoint improvement in beta cell function as assessed by HOMAB. HOMA-B increased from a mean $( \pm$ SEM $) 48.8 \pm 2.0 \%$ at baseline to a mean $67.6 \pm 3.2 \%$ at endpoint $(p<0.001)$.
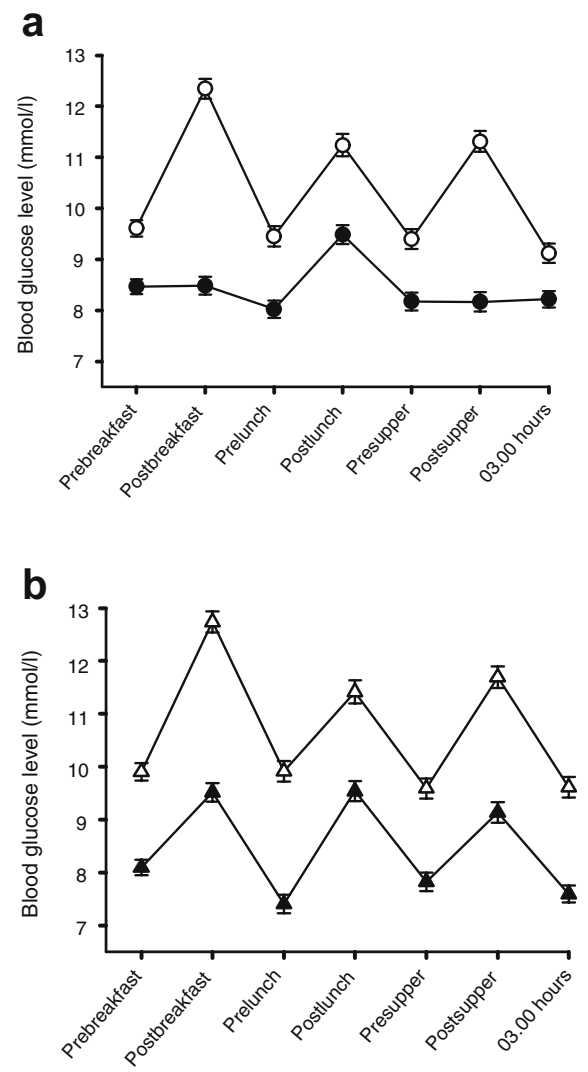

Fig. 3 Seven-point self-monitored blood glucose profiles. Least-squares mean (SEM) data for self-monitored blood glucose concentrations at baseline and week 52 are shown for exenatide (open circles, week 0; closed circles, week 52) and premixed insulin (open triangles, week 0; closed triangles, week 52) groups
HOMA-S did not change significantly among exenatidetreated patients.

Safety and clinical laboratory findings The incidence of gastrointestinal adverse events was higher with exenatide than with premixed insulin (Table 3). Nausea (33\% incidence during 52 weeks) and vomiting (15\% incidence) were mostly mild or moderate in severity. Together, $5.1 \%$ of patients withdrew because of gastrointestinal-related adverse events. Regarding anti-exenatide antibody status, 107 (45\%) patients were antibody-positive and 131 (55\%) patients were antibody-negative. Adverse events emerging from the treatment were not found to be associated with immune reactions to anti-exenatide antibodies and no significant association was observed between anti-exenatide antibody status and change in $\mathrm{HbA}_{1 \mathrm{c}}$ or fasting serum glucose.

HDL-cholesterol increased to a greater degree in the premixed insulin group (least-squares mean \pm SEM difference, exenatide minus insulin, $-0.04 \pm 0.01 \mathrm{mmol} / 1 ; p=0.003$ ); no additional significant baseline-to-endpoint changes in fasting lipid parameters were observed. No other clinically meaningful changes potentially attributable to exenatide treatment were identified from clinical chemistry and haematology assessments. A statistically significant mean reduction in both systolic blood pressure $(-5 \pm 15 \mathrm{mmHg}, p<0.001)$ and diastolic blood pressure $(-2 \pm 10 \mathrm{mmHg}, p=0.03)$ was observed at endpoint in the exenatide group. Blood pressure did not change significantly with premixed insulin (systolic blood pressure $\Delta 1 \pm 16 \mathrm{mmHg}, p=\mathrm{NS}$; diastolic blood pressure $\Delta 1 \pm 10 \mathrm{mmHg}, p=\mathrm{NS})$.

The overall hypoglycaemia rates were similar across treatment groups at endpoint (least-squares mean \pm SEM: exenatide $4.7 \pm 0.7$ events/patient-year, premixed insulin 5.6 \pm 0.7 events/patient-year). No severe hypoglycaemia was reported during the study. Although the incidence (i.e. number of patients experiencing at least one event) of nocturnal hypoglycaemia was significantly lower in the exenatide group at endpoint (exenatide 17\% [44/253], premixed insulin 25\% [62/248]; $p<0.038$ ), an analysis of rates adjusted for baseline 
Table 3 Overview of adverse events

\begin{tabular}{lll}
\hline Adverse event & \multicolumn{2}{l}{ Patients $(\%)$} \\
\cline { 2 - 3 } & $\begin{array}{l}\text { Exenatide } \\
(n=253)\end{array}$ & $\begin{array}{l}\text { Premixed insulin } \\
(n=248)\end{array}$ \\
\hline TEAEs & $179(70.8)$ & $123(49.6)$ \\
TEAEs occurring in $\geq 2 \%$ of patients & \\
Nausea & $84(33.2)$ & $1(0.4)$ \\
Vomiting & $38(15.0)$ & $8(3.2)$ \\
Nasopharyngitis & $28(11.1)$ & $24(9.7)$ \\
Diarrhoea & $24(9.5)$ & $5(2.0)$ \\
Influenza & $18(7.1)$ & $16(6.5)$ \\
Headache & $12(4.7)$ & $13(5.2)$ \\
Back pain & $11(4.3)$ & $10(4.0)$ \\
Anorexia & $7(2.8)$ & $0(0.0)$ \\
Dyspepsia & $7(2.8)$ & $1(0.4)$ \\
Arthralgia & $6(2.4)$ & $4(1.6)$ \\
Bronchitis & $6(2.4)$ & $6(2.4)$ \\
Depression & $6(2.4)$ & $1(0.4)$ \\
Pain in extremity & $6(2.4)$ & $8(3.2)$ \\
Other clinically relevant TEAEs & $34(13.4)$ & $16(6.4)$ \\
Anorexia/decreased appetite & $11(4.3)$ & $0(0.0)$ \\
Injection site reactions & $4(1.6)$ & $1(0.4)$ \\
Cardiac disorders & $10(4.0)$ & $5(2.0)$ \\
Hypertension & $5(2.0)$ & $7(2.8)$ \\
Neoplasms & $1(0.4)$ & $2(0.8)$ \\
Accidental falls & $3(1.2)$ & $1(0.4)$ \\
Serious adverse events & $19(7.5)$ & $11(4.4)$ \\
Deaths & $2(0.8)$ & $1(0.4)$ \\
\hline & & \\
& &
\end{tabular}

Hypoglycaemic events are not shown. Patients may be counted in more than one category. None of the serious adverse events resulting in death were considered related to study drug. Cardiac disorders included: angina pectoris, myocardial infarction, atrial fibrillation, coronary artery disease, acute coronary syndrome, atrial flutter and bundle branch block left

TEAE treatment-emergent adverse event

$\mathrm{HbA}_{1 \mathrm{c}}$ and country revealed that neither daytime (exenatide: $4.1 \pm 0.6$ events per patient-year, premixed insulin $4.4 \pm 0.7$ events/patient-year) nor nocturnal (exenatide: $0.6 \pm 0.2$ events per patient-year, premixed insulin $1.1 \pm 0.2$ events/patientyear) hypoglycaemia rates were significantly different between groups at endpoint. The overall hypoglycaemia rates were decreased following sulfonylurea dose reductions in exenatide-treated patients (mean $\pm \mathrm{SD}$ : before sulfonylurea reduction, $26.9 \pm 43.3$ events/patient-year; after sulfonylurea reduction, $6.1 \pm 8.3$ events per patient-year).

\section{Discussion}

This 52-week comparator trial demonstrates that exenatide twice daily has a similar glycaemic effect to premixed insulin aspart, as demonstrated by the reduction in $\mathrm{HbA}_{1 \mathrm{c}}$ values (Fig. 2, Table 2). The current study is the longest controlled study involving exenatide and presents results consistent with the previous comparator trial (insulin glargine) [12], as well as the 30-week placebo-controlled trials and their open-label extensions [8-11]. The aforementioned consistency is most apparent regarding the progressive weight reduction with exenatide (Fig. 2), in contrast to the weight increase observed with insulin regimens in this and earlier studies [12, 17-20] The findings of recent insulin comparator trials are of particular importance, because starting insulin treatment with longacting insulin preparations, as studied by Heine et al. [12], or premixed insulin preparations are currently the recommended next steps in the escalation of glucose-lowering therapy in type 2 diabetic patients who have suboptimal metabolic control on oral glucose-lowering agents [21].

Exenatide is thought to be a possible alternative to initiating treatment with insulin, possessing properties that clearly differ from those of insulin. In the present study, glycaemic increments and peak glucose concentrations after breakfast and supper, the two meals before which exenatide/ insulin were injected, were significantly improved with exenatide (Fig. 3), indicating substantially better postprandial control. Postprandial glucose control is recognised as an important contributor to overall glycaemic control [22, 23] and the risk of diabetic complications [24]. Premixed insulins are known to provide convenient mealtime dosing and coverage for both fasting and postprandial glucose [25, 26]. Interestingly, the results of the current study suggest that exenatide is as effective as premixed insulin for fasting glucose control, but superior with respect to postprandial control. These data provide additional information, which may enhance clinical decision-making (exenatide or insulins) for patients unable to achieve appropriate glycaemic control with metformin and sulfonylurea.

Insulin dose titration was guided by glucose selfmonitoring and adjustments were made during repeated contacts between patients and their physicians. Although dose titration potentially allows more flexibility for optimising treatment, standard twice-daily dosing is a convenient feature of exenatide treatment. The question arises of whether the titration of insulin doses should have been more aggressive, potentially leading to better glycaemic control. Investigators in the current trial were encouraged to titrate insulin doses as high as clinically possible, yet the daily insulin doses at endpoint, as well as the proportion of patients who achieved a target $\mathrm{HbA}_{1 \mathrm{c}}$ of $\leq 7.0 \%$, were generally lower than in previous studies of premixed insulin preparations [20, 26-28]. In addition to biases intrinsic to open-label studies [29], multiple factors could have influenced the comparatively low endpoint mean insulin dose observed in this trial. For example, a forced titration schedule was not used in this trial; rather, investigators were instructed to use fasting and postprandial glycaemic targets as a guide, while seeking to achieve an optimal 
balance between glycaemic control and risk of hypoglycaemia as dictated by best clinical practice. In addition, a fear of hypoglycaemic episodes or pronounced increases in body weight may have precluded the use of higher insulin doses. It should also be considered that all patients in the current trial remained on both metformin and sulfonylurea, whereas in the previous premixed insulin trials, metformin and sulfonylurea therapy were stopped [27] or only metformin was continued $[20,26,28]$.

Certainly, a notable strength of the present study is the long duration. The use of exenatide and premixed insulin should reflect their real-world use closely, especially with respect to the potentially conservative insulin dose titration mentioned above. Nevertheless, premixed insulin may be a most adequate comparator for exenatide because both agents are injected subcutaneously twice daily at the same times, and both have the potential to favourably influence fasting and postprandial glucose.

In line with recent clinical trials [8-12] with exenatide, there was a substantial and progressive decrease in body weight (Fig. 2) associated with its use, in contrast to the weight gain commonly observed with initiating insulin treatment [20]. Interestingly, $\mathrm{HbA}_{1 \mathrm{c}}$ was not further reduced between weeks 16 and 52 (Fig. 2), despite a reduction in body weight during the same period (Fig. 2), indicating that the observed weight reduction was not sufficient to have a detectable impact on glycaemic control. It should be noted that, similarly to findings from the 82-week open-label extension of the placebo-controlled trials [11], weight reduction occurred to a similar magnitude in groups with and without gastrointestinal adverse events. Thus, it appears that gastrointestinal side effects were not a significant cause of the weight reduction observed in patients receiving exenatide. Rather, exenatide may influence weight loss mainly through energy restriction by a mechanism similar to GLP-1 [30, 31], i.e. 2 centrally mediated regulation of appetite, satiety and food intake. It may therefore be associated with health benefits similar to those of a diet therapy. Recent data from open-label extension trials with exenatide [32] indicate that weight loss with exenatide remains progressive for up to 2 years. It should be noted that the long-term implications of progressive weight reduction for exenatide-treated patients, with respect to glycaemic control and overall systemic effects, have not been fully elucidated, especially given that incretin mimetics are the first available therapeutic class shown to have such effects. However, given the central role of obesity in the pathophysiology of type 2 diabetes [33], and the development of cardiovascular complications through obesity-associated risk factors [34-38], the availability of glucose-lowering agents that also prevent further increases of these risk factors could be a therapeutic advance and of particular value to patients chronically struggling to lose weight.
The overall safety profile of exenatide observed in the present trial did not differ substantially from previous trials [8-12] and the withdrawal rate in this trial was similar to the rate observed in the previously reported exenatide vs insulin (glargine) comparator trial [12]. Adverse gastrointestinal events occurred more frequently with exenatide than with premixed insulin, in up to one-third of the patients. Most of these episodes were mild or moderate in severity, transient, and rarely $(5.1 \%)$ led to discontinuation of exenatide treatment. Hypoglycaemia occurred mostly during daytime and none of the episodes was severe. With a background of the combined use of metformin and sulfonylurea, the occurrence of hypoglycaemic episodes was not unexpected with either insulin or exenatide. Exenatide alone or in combination with metformin does not increase the risk of hypoglycaemia [9]. In the current study, the role of sulfonylurea in eliciting hypoglycaemia is supported by the fact that the number of hypoglycaemic episodes tended to be less when the dose of sulfonylurea was reduced. Therefore, the safety of exenatide may be increased by preferentially combining it with agents that do not provoke hypoglycaemia. On the other hand, with insulin, the likelihood of hypoglycaemia varies inversely with the degree of metabolic control, and probably would be increased by further intensifying treatment.

In summary, exenatide therapy may be an alternative to initiation of insulin therapy, when oral glucose-lowering agents fail to maintain adequate glycaemic control. The reason for this is that exenatide provides overall glycaemic control comparable to premixed insulin, and has the added benefit of weight reduction. The present results should encourage large-scale exenatide studies designed to detect potential influences on health outcomes, such as clinical endpoints related to micro- and macrovascular consequences of type 2 diabetes. For example, the blood pressure reduction seen in this trial is consistent with data from the 82-week open-label extension trial [11], where exenatide was associated with a significant reduction in diastolic blood pressure $(-2.7 \mathrm{mmHg})$. Further study of this phenomenon and its potential impact on the progression of diabetes and its complications is warranted [39].

Acknowledgements We wish to thank the patients, investigators, and their staff for participating in the study. We also wish to thank the following for providing technical and material support: N. Yurgin, K. Gladkov, J. Burger, J. Reviriego, M. Mihm.

Duality of interest M. A. Nauck is a consultant for Eli Lilly and Company and Amylin Pharmaceuticals, has received grant support for clinical studies involving exenatide, as well as honoraria for lectures, and was invited to present the findings of this trial at congresses. S. Duran is a clinical trial investigator for Eli Lilly and Company and Amylin Pharmaceuticals. M. Trautmann, A. Festa, R. Brodows, D. Johns and J. Northrup are employees and/or stockholders of Eli Lilly and Company. D. Kim is an employee and stockholder of Amylin 
Pharmaceuticals. Eli Lilly and Company and Amylin Pharmaceuticals are involved in the development of exenatide.

\section{References}

1. Drucker DJ (2003) Enhancing incretin action for the treatment of type 2 diabetes. Diabetes Care 26:2929-2940

2. Kendall DM, Kim D, Maggs D (2006) Incretin mimetics and dipeptidyl peptidase-IV inhibitors: a review of emerging therapies for type 2 diabetes. Diabetes Technol Ther 8:385-396

3. Joy SV, Rodgers PT, Scates AC (2005) Incretin mimetics as emerging treatments for type 2 diabetes. Ann Pharmacother 39:110-118

4. Nielsen LL, Young AA, Parkes DG (2004) Pharmacology of exenatide (synthetic exendin-4): a potential therapeutic for improved glycemic control of type 2 diabetes. Regul Pept 117:77-88

5. Keating GM (2005) Exenatide. Drugs 65:1681-1692

6. Tourrel C, Bailbe D, Meile MJ et al (2001) Glucagon-like peptide1 and exendin- 4 stimulate beta-cell neogenesis in streptozotocintreated newborn rats resulting in persistently improved glucose homeostasis at adult age. Diabetes 50:1562-1570

7. Xu G, Stoffers DA, Habener JF, Bonner-Weir S (1999) Exendin-4 stimulates both beta-cell replication and neogenesis, resulting in increased beta-cell mass and improved glucose tolerance in diabetic rats. Diabetes 48:2270-2276

8. Buse JB, Henry RR, Han J et al (2004) Effects of exenatide (exendin-4) on glycemic control over 30 weeks in sulfonylureatreated patients with type 2 diabetes. Diabetes Care 27:2628-2635

9. DeFronzo RA, Ratner RE, Han J et al (2005) Effects of exenatide (exendin-4) on glycemic control and weight over 30 weeks in metformin-treated patients with type 2 diabetes. Diabetes Care 28:1092-1100

10. Kendall DM, Riddle MC, Rosenstock J et al (2005) Effects of exenatide (exendin-4) on glycemic control over 30 weeks in patients with type 2 diabetes treated with metformin and a sulfonylurea. Diabetes Care 28:1083-1091

11. Blonde L, Klein EJ, Han J et al (2006) Interim analysis of the effects of exenatide treatment on A1c, weight and cardiovascular risk factors over 82 weeks in 314 overweight patients with type 2 diabetes. Diabetes Obes Metab 8:436-447

12. Heine RJ, Van Gaal LF, Johns D et al (2005) Exenatide versus insulin glargine in patients with suboptimally controlled type 2 diabetes: a randomized trial. Ann Intern Med 143:559-569

13. Levy JC, Matthews DR, Hermans MP (1998) Correct homeostasis model assessment (HOMA) evaluation uses the computer program. Diabetes Care 21:2191-2192d

14. Wallace TM, Levy JC, Matthews DR (2004) Use and abuse of HOMA modeling. Diabetes Care 27:1487-1495

15. Fineman MS, Bicsak TA, Shen LZ et al (2003) Effect on glycemic control of exenatide (synthetic exendin-4) additive to existing metformin and/or sulfonylurea treatment in patients with type 2 diabetes. Diabetes Care 26:2370-2377

16. Piaggio G, Elbourne DR, Altman DG et al (2006) Reporting of noninferiority and equivalence randomized trials: an extension of the CONSORT statement. JAMA 295:1152-1160

17. UK Prospective Diabetes Study Group (1995) Overview of 6 years' therapy of type II diabetes: a progressive disease. UK Prospective Diabetes Study 16. Diabetes 44:1249-1258

18. UK Prospective Diabetes Study (UKPDS) Group (1998) Intensive blood-glucose control with sulphonylureas or insulin compared with conventional treatment and risk of complications in patients with type 2 diabetes (UKPDS 33). Lancet 352:837-853

19. UK Prospective Diabetes Study (UKPDS) Group (1998) Effect of intensive blood-glucose control with metformin on complications in overweight patients with type 2 diabetes (UKPDS 34). Lancet 352:854-865

20. Raskin P, Allen E, Hollander $P$ et al (2005) Initiating insulin therapy in type 2 diabetes: a comparison of biphasic and basal insulin analogs. Diabetes Care 28:260-265

21. IDF Clinical Guidelines Task Force (2005) Global guideline for Type 2 diabetes. International Diabetes Federation, Brussels

22. Bastyr EJ III, Stuart CA, Brodows RG et al (2000) Therapy focused on lowering postprandial glucose, not fasting glucose, may be superior for lowering HbA1c. IOEZ Study Group. Diabetes Care 23:1236-1241

23. de Veciana M, Major CA, Morgan MA et al (1995) Postprandial versus preprandial blood glucose monitoring in women with gestational diabetes mellitus requiring insulin therapy. N Engl J Med 333:1237-1241

24. Monnier L, Lapinski H, Colette C (2003) Contributions of fasting and postprandial plasma glucose increments to the overall diurnal hyperglycemia of type 2 diabetic patients: variations with increasing levels of HbA1c. Diabetes Care 26:881-885

25. Garber AJ (2006) Premixed insulin analogues for the treatment of diabetes mellitus. Drugs 66:31-49

26. Malone JK, Bai S, Campaigne BN et al (2005) Twice-daily premixed insulin rather than basal insulin therapy alone results in better overall glycaemic control in patients with Type 2 diabetes. Diabet Med 22:374-381

27. Janka HU, Plewe G, Riddle MC et al (2005) Comparison of basal insulin added to oral agents versus twice-daily premixed insulin as initial insulin therapy for type 2 diabetes. Diabetes Care 28:254-259

28. Schwartz S, Sievers R, Strange P et al (2003) Insulin 70/30 mix plus metformin versus triple oral therapy in the treatment of type 2 diabetes after failure of two oral drugs: efficacy, safety, and cost analysis. Diabetes Care 26:2238-2243

29. Altman D, Schulz K, Moher D et al (2001) The revised CONSORT statement for reporting randomized trials: Explanation and elaboration. Ann Intern Med 134:663-694

30. Flint A, Raben A, Astrup A, Holst JJ (1998) Glucagon-like peptide 1 promotes satiety and suppresses energy intake in humans. J Clin Invest 101:515-520

31. Turton MD, O'Shea D, Gunn I et al (1996) A role for glucagon-like peptide-1 in the central regulation of feeding. Nature 379:69-72

32. Henry RR, Ratner RE, Stonehouse AH et al (2006) Exenatide maintained glycemic control with associated weight reduction over two years in patients with type 2 diabetes. [Abstract]. Diabetes 55:A116

33. Colditz GA, Willett WC, Rotnitzky A, Manson JE (1995) Weight gain as a risk factor for clinical diabetes mellitus in women. Ann Intern Med 122:481-486

34. Goldstein DJ (1992) Beneficial health effects of modest weight loss. Int J Obes Relat Metab Disord 16:397-415

35. Janssen I, Katzmarzyk PT, Ross R (2004) Waist circumference and not body mass index explains obesity-related health risk. Am J Clin Nutr 79:379-384

36. Mokdad AH, Ford ES, Bowman BA et al (2003) Prevalence of obesity, diabetes, and obesity-related health risk factors, 2001. JAMA 289:76-79

37. Van Gaal LF, Wauters MA, De Leeuw IH (1997) The beneficial effects of modest weight loss on cardiovascular risk factors. Int J Obes Relat Metab Disord 21 (Suppl 1):S5-S9

38. Zhu S, Wang Z, Heshka S et al (2002) Waist circumference and obesity-associated risk factors among whites in the third National Health and Nutrition Examination Survey: clinical action thresholds. Am J Clin Nutr 76:743-749

39. UK Prospective Diabetes Study Group (1998) Tight blood pressure control and risk of macrovascular and microvascular complications in type 2 diabetes: UKPDS 38. BMJ 317:703-713 